\title{
EFFECT OF SMART STRUCTURES ON AERODYNAMIC PERFORMANCE OF HORIZONTAL AXIS WIND TURBINE
}

\author{
Hira Syed \\ Department of Industrial Engineering \\ University of Management and Technology \\ Lahore, Pakistan
}

\author{
Dr.Gulraiz Ahmed \\ Department of Mechanical Engineering \\ University of Central Punjab, Lahore, Pakistan
}

\begin{abstract}
In renewable energy the wind energy is the most significant source. The wind turbine suppresses the kinetic energy of the wind. Current research focuses on improving the aerodynamic performance of wind turbine blades through wind tunnel tests and theoretical studies. These exercises are time taking and require considerable laboratory resources. Similarly, simulation of wind turbines using CFD software (Computational Fluid Dynamics) provides cost-effective solutions for aerodynamic analysis of the blades. Due to the energy crisis in Pakistan, we need a solution to overcome the power shortage. Wind energy is an economical and affordable energy. In this study, two-dimensional airfoil S4310, was selected for the blade cross section. $2.1 \mathrm{~m}$ cord length from root and $0.67 \mathrm{~m}$ cord length from tip of the blade, aerodynamic analysis of this model was performed using ANSYS-FLUENT software. Using the $k-\varepsilon$ turbulence model, the lift and drag coefficients were computed for wind-turbine blade at $0^{\circ}-14^{\circ}$ angles of attack (AOA). The CFD results accomplish by all together solving momentum ,continuity and the Navier-Stokes equations using a standard non-linear $k-\varepsilon$ solver. The smart structures were also applied on the wing in which active twist was applied to the blade using twist angles from $0^{\circ}-10^{\circ}$ and similarly the lift to drag ratio were considered.
\end{abstract}

Keywords - Computational Fluid Dynamics (CFD), Angle of Attack (AOA), Horizontal Axis Wind Turbine(HAWT), Angle of Attack (AOA)

\section{INTRODUCTION}

In recent years it has been observed that energy utilization which is an insufficient availability and extremely increasing the need for the consumption of uncontaminated renewable energy sources. Owing to a rise in prices of oil and human understanding about technology it encourages for renewable energy sources. In renewable energy resources the wind energy is considered is one of the good source of energy. In present technology, the wind energy which is low cost is more capable sources of renewable energy as compared to coal which is more predictable source of energy [1]. Due to energy crisis in Pakistan, we have to produce an economical, environment friendly source of energy which can bring out the deficiency from the crisis and wind turbine is the best source of energy which gets the energy from the wind.

When we talk about renewable resources the wind energy is expensive resource of energy as compared to other renewable energy sources [2]. Similarly, if solar energy is considered, and we talk about solar energy, that really affected by climate and weather, due to increase in temperature it disturbs the ecosystem and plants reproduction and it also effects on the glaciers specially on the polar side region. Extraction of energy from the wind turbine was formally invented by the engineers. It is the best source of energy that a wind converts into the useful electric energy, usually under this process a turbine is used. There are four main parts of wind turbine which is called rotor, generator, motorized chain and control system. The wind drives the rotor and it's rotates at definite speed with relating to the speed of wind, due to the different parameters of control system the generator generates the electrical energy

\subsection{Types of Wind Turbine}

There are two types of Wind turbines which are commonly known as horizontal and vertical axis. A horizontal axis wind turbine contains its blades revolving around the axis parallel to the ground. The turbine in which its blades revolves around the axis at right angle to the ground is called vertical axis wind turbine as shown in Figure 1(a). There are a number of available designs for both types of wind turbines which have their own certain advantages and drawbacks. Although, the vertical axis wind turbine is very few available in market as compared to horizontal axis wind turbine. The size of wind turbines varies according to power requirements.

Length of the blades is the core part in describing and attaining the amount of electricity gets from the wind turbine. Small wind turbines can power to home having an electricity generating requirements of 10 kilowatts $(\mathrm{KW})$. Operationally largest wind turbines having capacity in operationally for generating of up to $10,000 \mathrm{KW}$ electricity. Large turbines are 
used to supply power to electric grid from the wind power plants and wind farms.
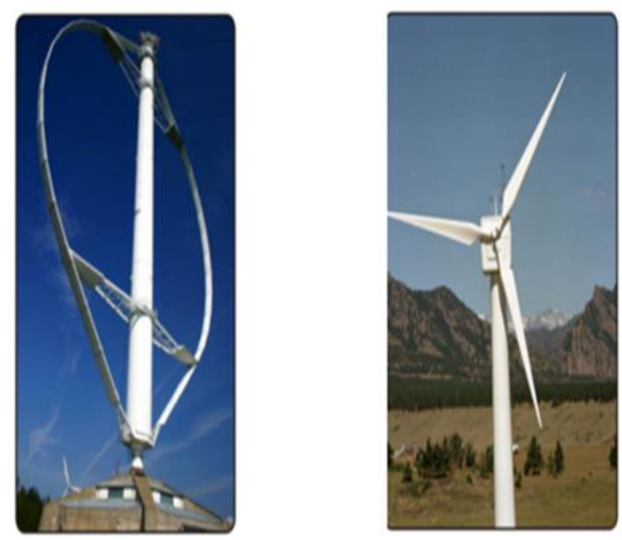

(a)Vertical axis wind turbine (b) Horizontal axis wind turbine

Figure 1: Wind Turbines

\subsubsection{Horizontal Axis Wind Turbine}

Horizontal axis wind turbines blades having resemblance with airplane propellers, which transmit the power by converting rotational motion into power and normally they have three big blades. The biggest horizontal-axis turbines are usually taller in length as 50-story buildings and usually having blades above 120 feet length. The advantage of higher turbines which generates the more electricity. Many wind turbines which are commonly used are horizontal-axis wind turbine. Horizontal axis wind turbine (HAWT) also called as lift-force wind turbine. The rotor axis place horizontally and the air is parallel to the rotor in horizontal axis wind turbine. The blades curve is a circular plane normal to the air flow, located upwind (in front of the tower) or downwind (behind the tower) as shown in Figure 1(b). The plus point of HAWT is the good aerodynamic competence and flexibility. Their main drawback is that all electricity equipment, rotor and gearing system place on rotor that is supported by tower, in addition the requirement of yawing to face the wind (U.S. Energy Information Administration)

\subsubsection{Parts of the Horizontal axis wind turbine}

1. The nacelle comprises off the main modules of the wind turbine that contains the gearbox, and the electrical generator, as shown in Figure 2.

2. Wind turbine tower support the nacelle and the rotor. Usually, it is a great advantage for having a high tower, when wind speeds increase more far away from the ground.

3. The rotor blades catch wind energy and pass on its power to the rotor hub.
4. Generator converted the mechanical energy of the rotating shaft into an electrical energy.

5. The Rotational speed of the shaft is increased by the gearbox for the generator.

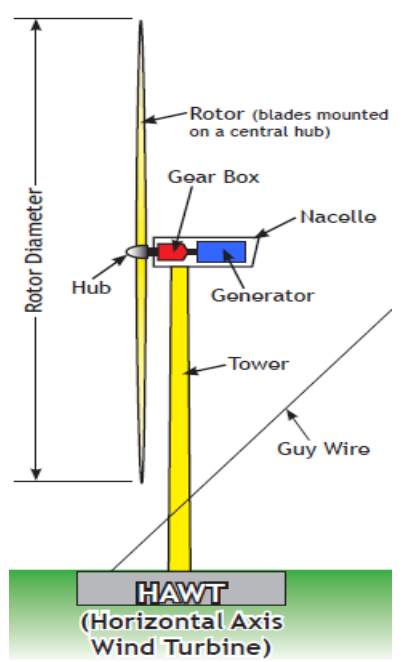

Figure2: Horizontal Axis Wind Turbine (HAWT)

\section{LITERATURE REVIEW}

\subsection{Wind Turbine Aerodynamics}

The main function of wind turbines is to produce energy using the wind. Therefore, the aerodynamics is an essential feature of wind turbines. All the wind turbines work for energy withdrawal idea. Even though the aerodynamic particulars are more dependent on a topology, but some topologies are applying for all types of turbine. Each one of the topology has an extreme level of control for a given flow, and some of the topologies are much enhanced than others. The method is used to extract power for strong force. On the whole, all turbines are classified on the basis of lift, or drag. Though lift based turbines are more effective, because of the amount of force that can be extracted from it.

\subsubsection{Lift and Drag coefficients}

Normally, there are one moment and two forces and that perform upon an airfoil, one is the lift force, second is the drag force and third is the moment force. The force is used to overcome the gravity is called Lift [3] and lift force is the force that is at right angle to direction of the air [4]. Lift is produced due to the result of when upper and lower surfaces of airfoil have uneven pressure on it. When the air direction is parallel to the force that force is called drag force [4]. Due to the viscous friction forces and uneven pressure on the airfoil surface the drag force is produced. If the lift force is greater the greater mass can be raised to the ground. For airfoil, lift to drag ratio must be maximum [2]. Therefore, if electricity produces from wind turbine the efficiency can be improved. 
Lift and drag coefficients $C_{L}$ and $C_{D}$ (Equations 2.1 and 2.2)

are given below:

$$
\begin{gathered}
C_{L}=\frac{F_{L}}{\frac{1}{2} \rho V_{0}^{2} c} \\
\frac{F_{L}}{\frac{1}{2} \rho V_{0}^{2} c}
\end{gathered}
$$

Where, $\rho$ is the air density and airfoil chord length is c. The

force per length $(\mathrm{N} / \mathrm{m})$ unit is used for lift and drag forces which is shown in Equations (2.1) and (2.2).

\subsubsection{Airfoil}

Airfoils becomes one of the most perplexing and misinterpreted feature of designing a wind turbine blade. Airplane airfoils performance features are not satisfactory for wind turbine airfoils and their thickness is not up to the mark. In aviation and wind-turbine airfoils both give benefits for extensive laminar flow and for low drag. In history, with the use of commercial wind turbines during 1980s it experienced objectionable performance features associated with NACA series airfoils. In early 1970s there are different NACA airfoils had been developed into the World war II. These NACA airfoils were developed for turbulent flows and they create problems and separation bubbles when they applied on wind turbines. These airfoils are not suitable for laminar flows. Due to the separation bubbles the large difference has been seen in airfoil performance in roughness [5]. Airfoil are better to produce more lift and less drag as compared to flat plate of the same area because of highly efficient lifting shape. In modern airfoils the turbulent flow shows on the entire upper surface of the turbine blade just previous to maximum lift.

\subsubsection{Number of Blades}

In HAWT the number of blades has a prominently effect on a performance. The most commonly used designed in wind turbines are two blades design. The three blade design are also famous. Small HAWT which have more than three blades are normally called low speed. In low speed turbine the greater torque is produce. In contrast, the same energy consumption can be acquired with two or three blades at low torque in high speed wind turbines [2].

\subsubsection{Angle of Twist}

The lift produce on the airfoil is actually due to the angle of attack which is in the direction of airflow. It is noted that the angle of attack is dependent on two factors one is the rotational speed and second is the wind speed at specific radius. The twist angle defined as the angle between the angle of attack of the airfoil and the tip speed ratio. The airfoil is kept angle at the hub due to the high ratio of wind speed to blade radial velocity. Apart from this the tip of the blade becomes normal to the wind [6].

\subsubsection{Angle of Attack}

The angle of attack is that angle approaching flow comparative to the cord line, in addition to this, all figures for $C_{L}$ and $C_{D}$ are mentioned comparative to this angle. If a single airfoil is used for the whole blade length then it would result in unsuitable design [7]. The airfoil sections have design accordingly on the blade because every section of the blade has its own relative air velocity and structure demand. For heavy load carried, the root of the blade has thick profile on the other hand the blade section has great minimum thickness

\subsubsection{Stall angle}

A stall is a situation where the angle of attack enhances away from a definite point in a manner when the lift starts to reduce. The angle at which this happens is called the critical angle of attack. A stall happens when the angle of attack of a blade or airfoil are so high that the airflow over the upper surface of the blade is apart from the blade, instead of remaining in touch to it, because of this the blade produce less lift and more drag, rendering it harder to maintain lift production. For many airfoils the stall angle lies between $15^{\circ}-20^{\circ}$.

\subsection{Smart Structures}

The smart structures are becoming more famous in recent research because they change their shape according to the wind direction. Along with this group smart blades can be design either control the aerodynamic surfaces or by applying the smart actuators [8].

Active twist is one of the method to design a smart structure in which the blade is twisted from the tip or twisting the entire blade over its complete extent, see Figure 3(a). Due to the twist the local angle of attack changed. At tip the variation in pitch is greater, which is functional for aerodynamic control. Due to the high variation in pitch the span wise twist is not preferable [8]. The idea is based on the dynamically controlled bending-torsion or tension-torsion pairing. From research it is shown that this concept is better for helicopter applications and it gives good aerodynamic controls but for large scale wind turbine blades some drawbacks can be observed.

In active flaps change in aerodynamic forces at any condition is easiest therefore this concept is becoming more famous, see Figure 3(b). The flaps can be used in different methods i.e. 
reducing fatigue, peak loads are one of the methods from which we can use flaps, Therefore, it shown that from the numerical and experimental results that the fatigue load decrease in trailing edge flaps [9]. Now a day, presently researchers have started to design an optimized blade using this concept of active flaps. In figure below, here is the optimal blade design using the active flap concept.



(a) Actice Twist

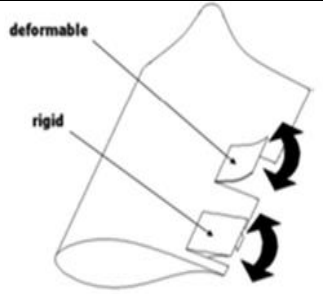

Figure 2.16: Active flap Concept (b) Active Flap
Fig 3 Smart Structures

\subsection{Computational Fluid Dynamics}

ANSYS FLUEN, STAR-CD are those CFD software which are mostly used in engineering. In all types of CFD software the main structure of Pre-Processer, Solver and Post-Processer are always present.

\subsubsection{The standard theories related to CFD}

For CFD simulation the main processes are the same regardless what CFD software is used. The formation of the equations is the compulsory for CFD modeling. There are three basic governing equations which are described below:

\section{Continuity Equation}

$\frac{D \rho}{D t}+\rho \frac{\partial U_{i}}{\partial x_{i}}$

\section{Momentum Equation}

$\rho \frac{\partial U_{j}}{\partial t}+\rho U_{i} \frac{\partial U_{j}}{\partial x_{i}}=-\frac{\partial P}{\partial x_{j}}-\frac{\partial \tau_{i j}}{\partial x_{i}}+\rho g_{j}$

In equation (2.4) the $\rho \frac{\partial U_{j}}{\partial t}$ is the local change with time, $\rho U_{i} \frac{\partial U_{j}}{\partial x_{i}}$ is the momentum convection, $\frac{\partial P}{\partial x_{j}}$ is the surface force, $\frac{\partial \tau_{i j}}{\partial x_{i}}$ is the molecular dependent momentum change (diffusion) and $\rho g_{j}$ is the mass force.

\section{Energy equation}

$$
\rho c_{\mu} \frac{\partial T}{\partial t}+\rho c_{\mu} U_{i} \frac{\partial T}{\partial x_{i}}=-P \frac{\partial U_{i}}{\partial x_{i}}+\lambda \frac{\partial^{2} T}{\partial x_{i}^{2}}-\tau_{i j} \frac{\partial U_{j}}{\partial x_{i}}
$$

In equation (2.5) where $\rho c_{\mu} \frac{\partial T}{\partial t}$ is the local change with time, $\rho c_{\mu} U_{i} \frac{\partial T}{\partial x_{i}}$ is the convection term, $P \frac{\partial U_{i}}{\partial x_{i}}$ is the pressure work, $\lambda \frac{\partial^{2} T}{\partial x_{i}^{2}}$ is the Heat flux (diffusion) and $\tau_{i j} \frac{\partial U_{j}}{\partial x_{i}}$ is the irreversible transfer of mechanical energy into heat. In this project the energy equation did not consider.

\subsubsection{Types of Mesh}

Single cell is the basic component in structured and unstructured mesh. In three-cornered and four-sided cells, the two-dimensional unstructured mesh is the central element. In structured mesh the rectangular cells are used [2]. For gaining the rational result the mesh quality plays an important role for simulation engineers. It is notice that if the nodes in the mesh are high the simulation takes more time to solve the solution but generating the efficient mesh is compulsory.

There are three different methods to solve the mesh in simulation one is finite different method, finite element method, and finite volume method. Fluent, CFX used finite volume method for simulation. If comparison is made between all three models FVM and FEM are easily calculated as compared to FVM for the distribution of unstructured mesh and it has a great flexibility for effective range of geometries

\subsubsection{Turbulence models}

In computational fluid dynamics software, turbulence flow models are used for wind turbines. The method of turbulence numerical simulation is actually containing two main parts that are Direct Numerical Simulation and Indirect Numerical Simulation.

Reynolds-Averaged Navier-Stokes (RANS)

For turbulence modeling the RANS is consider the oldest and most common technique. The Reynolds-Averaged NavierStokes equation is given below:

$\rho \frac{D U_{i}}{D t} \quad=\quad \frac{\partial P}{\partial X_{i}}$

$+\frac{\partial}{\partial X_{i}}\left[\mu\left(\frac{\partial U_{i}}{\partial x_{j}}+\frac{\partial U_{j}}{\partial x_{i}}\right)-\rho \overline{u_{i}^{I} u_{j}^{I}}\right]$

The $\rho \frac{D U_{i}}{D t}$ term in equation (2.6) is called the change in mean momentum and in equation (2.6) the right hand side is called the divergence stress, $\rho \overline{u_{\imath}^{\prime} u_{\jmath}^{\prime}}$ is the Reynolds stresses. In turbulence model there are one equation and two equations models are present. One equation model is "Spalart-Allmaras" and "standard $\mathrm{k}-\varepsilon$ " is the two equation model which are used mostly in many CFD software.

$\underline{\boldsymbol{k}}-\boldsymbol{\varepsilon}$ Model

In $\mathrm{k}-\varepsilon$ model, there are two transported variables one is the turbulence kinetic energy, $\mathrm{k}$ and the second one is turbulence 
dissipation, $\varepsilon$. In standard $\mathrm{k}-\varepsilon$ model, the turbulence kinetic energy (k) are shown in equation 2.8 :

$$
\begin{aligned}
& \frac{\partial}{\partial_{t}}(\rho k)+\frac{\partial}{\partial x_{i}}\left(\rho k u_{i}\right)=\frac{\partial}{\partial x_{j}}\left[\left(\mu+\frac{\mu_{t}}{\sigma_{k}}\right) \frac{\partial_{k}}{\partial x_{j}}\right]+ \\
& P_{k}+P_{b}-\rho \varepsilon-Y_{M}+S_{k}
\end{aligned}
$$

The turbulence dissipation $\varepsilon$ can be described in equation 2.9:

$\frac{\partial}{\partial_{t}}(\rho \varepsilon)+\frac{\partial}{\partial x_{i}}\left(\rho \varepsilon u_{i}\right)=\frac{\partial}{\partial x_{i}}\left[\left(\mu+\frac{\mu_{t}}{\sigma_{s}}\right)\right.$

$\left.\frac{\partial \varepsilon}{\partial x_{i}}\right]+C_{1 s} \frac{\varepsilon}{k}\left(G_{k}+C_{3 s} G_{b}\right)-C_{2 s} \rho \frac{\epsilon^{2}}{k}+S_{s}$

The $\mu_{t}$ is given by in equation 2.10 :

$\mu_{t}=\rho C_{\mu} \frac{k^{2}}{\varepsilon}$

The $P_{k}$ is given by in equation 2.11 :

$P_{k}=\mu_{t} S^{2}$

In these equations, $G_{k}$ and $G_{b}$ are the formations of turbulence kinetic energy. $Y_{M}$ is the dilatation dissipation term. The $C_{1 \varepsilon}, C_{2 \varepsilon}, C_{\mu}, \sigma_{\varepsilon}$ and $\sigma_{k}$ are the constant which values are $1.44,1.92,0.09,1.3,1$ respectively. In fluent, $S_{\varepsilon}$ and $S_{k}$ are the user-defined terms.

\section{METHODOLOGY}

\subsection{Selection of an Airfoil}

The selection of an Airfoil is from the site UIUC [10]. The S4310 Airfoil has been chosen for the wing. The data points of the Airfoil from the website UIUC. It is the applied aerodynamic group, Department of aerospace engineering, University of Illinois in United States. The X and Y coordinate file is scaled up to 2.1 and 0.67 from root to tip respectively according to our requirement in MATLAB. After scaling the coordinate file, they are imported into ANSYS, where three dimensional curve is sketched from data points of the airfoil. After import the coordinate file the skin/loft command is used for the blade. In Figure 4 the S4310 airfoil has been shown.

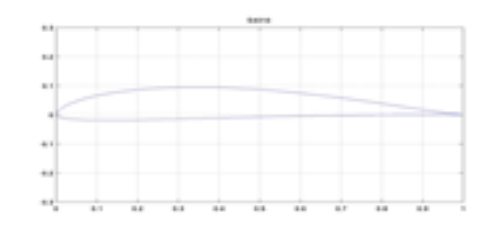

Figure 4: 54310 airfoil section

\subsection{Design of a Blade}

The dimensions are taken from the article for $1 \mathrm{MW}$ wind turbine with $0.44 \mathrm{~kW} / \mathrm{m}^{2}[11]$. The total length of the blade is selected to be $30 \mathrm{~m}$. The root cord length is $2.1 \mathrm{~m}$ and the tip cord length is $0.67 \mathrm{~m}$. The root to tip ratio is 3.13 . The selection of an airfoil is from the UIUC site which is American institute of aerospace [10]. In Figure 5 the design of a blade is shown.

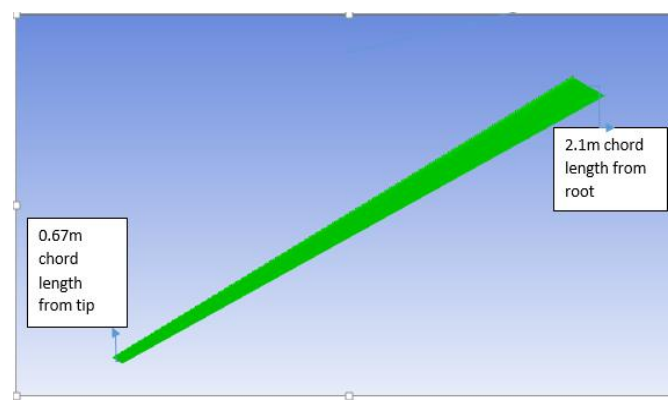

Figure 5: Schematic diagram showing the created blade

\subsection{Computational Domain}

The computational domain is created around the wing for the CFD analysis. After this the circle of $50 \mathrm{~m}$ radius was drawn on the XY axis of the wing. In design modular the revolve command was used for $90^{\circ}$, then for completion of domain the extrusion of $30 \mathrm{~m}$ was done. After that named selections for different portions of the computational domain were performed. Such as inlet, outlet, sides, symmetry and wing as shown in Figure 6.



Figure 6: Schematic diagram of computational domain

\subsection{MESHING}

The most important step after defining the computational domain is the development of mesh in preprocess. To solve the flow physics within the domain CFD needs the subdivision of the domain into a number of smaller, non-over projecting sub domain, as a result the formation of mesh covers the entire domain. The triangular mesh was considered because the geometry was simple and it is suitable for simple geometries. 


\section{International Journal of Engineering Applied Sciences and Technology, 2021 \\ Vol. 5, Issue 11, ISSN No. 2455-2143, Pages 62-71 \\ Published Online March 2021 in IJEAST (http://www.ijeast.com)}

There are three types of mesh that had been created the coarse mesh, the medium mesh and the fine mesh.

\subsubsection{Coarse Mesh}

In coarse mesh, in face sizing the number of division was 50 and in edge sizing the number of elements was (0.07). In the statistics number of elements were 708216 and the number of nodes were 124255. In Figure 7 the coarse mesh has been shown.



Figure 7: Coarse Mesh

\subsubsection{Medium Mesh}

In Medium Mesh, in face sizing the number of divisions was 100 and in the edge sizing the number of elements was (0.05). In the statistics bar the number of elements was1796344and the number of nodes was 324680.In Figure 8 the medium mesh is shown.

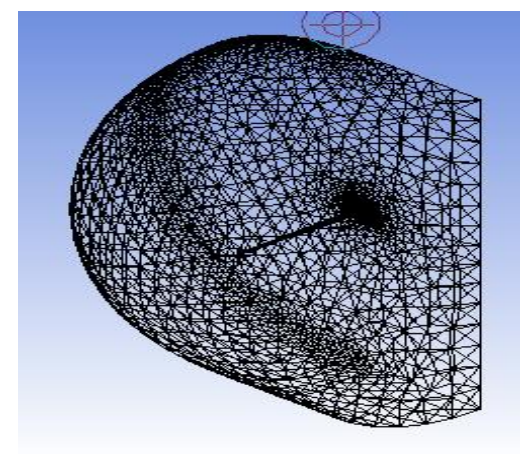

Figure 8: Medium Mesh

\subsubsection{Fine mesh}

In Fine mesh, in face sizing the number of divisions was 150 and in the edge sizing the number of elements was 0.03. In the statistics bar the number of elements was 1813069 and the number of nodes was 327969. In Figure 9 the fine mesh is shown.

In medium and fine mesh as we can see that the number of elements and the number of nodes both are not same. The mesh is supposed to be converged when further mesh refinement produces a negligible change in the solution so the mesh convergence was happened, so in this project the medium mesh is going to be considered and all the result are obtained from the medium mesh.

\subsection{Fluent Simulation}

The geometry and mesh are imported into FLUENT by default it has a single precision, a precise solution is required for these types of simulations. The residuals for the turbulence model was set to 0.001 . The iteration process could be stopped if the $C_{L}$ or $C_{D}$ seems to have stabilized, i.e. no further change was observed. Before starting a simulation in fluent, the mesh has to be checked and scaled for highly skewed elements, boundary conditions and initial conditions was also defined.

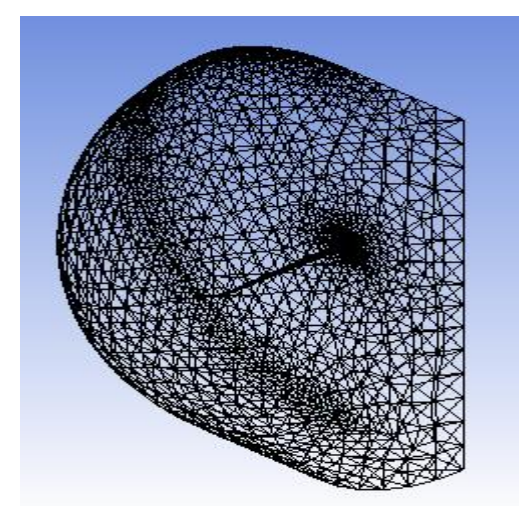

Figure 9 Fine Mesh

This also includes the compressibility, viscosity, laminar or turbulent flow, steady or time dependent flow.

\subsection{Initial Boundary Condition}

In the computational flow domain, in initial boundary condition the inlet velocity was $9 \mathrm{~m} / \mathrm{s}$ without given any angle of attack. The value of inlet velocity was taken from the Karachi area which was the monthly average wind speed from 2002-2005 as shown in table 3.1 [12].

\section{Table 3.1:-Average wind speed in Karachi}

\begin{tabular}{|c|c|c|c|c|c|c|c|c|c|}
\hline \multirow{2}{*}{\begin{tabular}{|c|} 
Table for \\
Fig-2 \\
\end{tabular}} & \multicolumn{9}{|c|}{ Monthly Average Wind Speed $(\mathrm{m} / \mathrm{s})$ at Karachi } \\
\hline & \multicolumn{3}{|c|}{ Karachi Apr2002 to Mar2003 } & \multicolumn{3}{|c|}{ Karachi Apr2003 to Mar2004 } & \multicolumn{3}{|c|}{ Karachi Apr2004 to Mar2005 } \\
\hline Months & AWS10m AI & AWS30m A & AWS50m & AWS $10 \mathrm{~m} \mathrm{~A}$ & AWS $30 \mathrm{~m}$ & AWS50m & AWS10m A & $30 \mathrm{~m} \mathrm{~A}$ & AWS50m \\
\hline Jan & 0.5 & 1.4 & 1.9 & 1.1 & 2.3 & 3. & 0.4 & 1.5 & 2.1 \\
\hline Feb & 0.7 & 1.8 & 2.3 & 0.6 & 1.7 & 2. & 0.9 & 2.2 & 2.8 \\
\hline Mar & 1.2 & 2.4 & 3.0 & 2.4 & 4.0 & 4. & 2.7 & 3.1 & 3.9 \\
\hline Apr & 1.9 & 3.6 & 4.5 & 1.7 & 3.2 & 4. & 4.3 & 6.2 & 7.2 \\
\hline May & 3.5 & 5.4 & 6.4 & 2.9 & 4.6 & 5. & 4.8 & 6.7 & 7.7 \\
\hline Jun & 3.1 & 4.8 & 5.8 & 3.2 & 4.9 & 5. & 6.3 & 7.7 & 9.0 \\
\hline Jul & 3.5 & 5.6 & 6.8 & 2.9 & 3.5 & 4. & 4.4 & 6.6 & 8.2 \\
\hline Aug & 2.3 & 3.7 & 4.5 & 2.8 & 4.1 & 5. & 2.6 & 5.8 & 7.5 \\
\hline Sep & 2.8 & 4.7 & 5.8 & 2.2 & 3.5 & 4. & 1.9 & 4.0 & 5.1 \\
\hline Oct & 0.5 & 0.9 & 1.1 & 0.4 & 1.2 & 1. & 1.3 & 2.4 & 3.1 \\
\hline Nov & 0.3 & 1.0 & 1.3 & 0.3 & 1.2 & 1. & 0.5 & 1.3 & 1.8 \\
\hline Dec & 0.5 & 1.4 & 1.9 & 0.6 & 1.8 & 2. & 0.5 & 2.1 & 3.0 \\
\hline Annual & 1.7 & 3.1 & 3.8 & 1.8 & 3.0 & 3. & 2.5 & 4.1 & 5.1 \\
\hline
\end{tabular}


In FLUENT, first the $\boldsymbol{k}-\boldsymbol{\varepsilon}$ model was choose. Second the boundary conditions were applied on the computational domain. There were six different regions which is shown on the boundary conditions that was inlet, outlet, symmetry, wing region, sides and interior region. For inlet, inlet velocity condition was set for outlet and sides, the pressure outlet condition was set and for symmetry the symmetry condition was set and for wing the wall condition was set at the end, for interior region the fluent automatically set to the interior condition.

\subsection{Discretization Scheme}

The discretization Scheme used for solving the $\mathrm{k}-\varepsilon$ model the different conditions are given in which pressure was standard, momentum was second order upwind and turbulence kinetic energy was also Second Order upwind. Turbulence Dissipation Rate was also Second Order upwind and the pressure velocity coupling was SIMPLE.

\section{RESUlTS}

\subsection{Angle of Attack Values}

In table 4.1 for different values of angle of attack blade shows different lift and drag values. In this research only lift coefficients are under observation because the blade is not an aero plane wing. It is a wind turbine blade so only lift forces are important and lift coefficients are considered. As in the table at $0^{\circ}$ angle the lift force is minimum and when the angle of attack is increased on the inlet velocity the lift coefficient increases gradually to $14^{\circ}$ the lift coefficient reaches its maximum value. The stall angle of the S4310 airfoil is $10^{\circ}$ so as, we can see that at angle $10^{\circ}$ the lift to drag ratio value is also at 10 , the blade reached its stall angle at 14 Degree.

Table 4.1:- $C_{L}, C_{D}$ and $C_{L} / C_{D}$ values for change in angle of attack

\begin{tabular}{|c|c|c|c|}
\hline $\begin{array}{c}\text { Angle of } \\
\text { Attack }\end{array}$ & $\begin{array}{c}\text { Lift } \\
\text { Coefficient } \\
\left(\boldsymbol{C}_{\boldsymbol{L}}\right)\end{array}$ & $\begin{array}{c}\text { Drag } \\
\text { Coefficient } \\
\left(\boldsymbol{C}_{\boldsymbol{D}}\right)\end{array}$ & $\begin{array}{c}\text { Lift /Drag } \\
\text { Ratio } \\
\left(\boldsymbol{C}_{L} / \boldsymbol{C}_{\boldsymbol{D}}\right)\end{array}$ \\
\hline $\begin{array}{c}\text { At angle } \\
\mathbf{0}^{\circ}\end{array}$ & 15.934 & 0.93276 & 17.08 \\
\hline $\begin{array}{c}\text { At angle } \\
\mathbf{2}^{\circ}\end{array}$ & 21.524 & 1.4749 & 14.6 \\
\hline $\begin{array}{c}\text { At angle } \\
\mathbf{4}^{\circ}\end{array}$ & 28.418533 & 1.9120603 & 14.8 \\
\hline
\end{tabular}

\begin{tabular}{|c|c|c|c|}
\hline $\begin{array}{c}\text { At angle } \\
\mathbf{8}^{\circ}\end{array}$ & 41.030582 & 3.3917565 & 12.09 \\
\hline $\begin{array}{c}\text { At angle } \\
\mathbf{1 0}^{\circ}\end{array}$ & 44.999971 & 4.415628 & 10.2 \\
\hline $\begin{array}{c}\text { At angle } \\
\mathbf{1 2}^{\circ}\end{array}$ & 46.773284 & 5.7793038 & 8.106 \\
\hline $\begin{array}{c}\text { At angle } \\
\mathbf{1 4}^{\circ}\end{array}$ & 46.840198 & 7.3550377 & 6.368 \\
\hline
\end{tabular}

\subsubsection{Effect of Angle of attack on Lift and drag coefficients}

In Figure 10(a) the behavior of lift coefficient against angle of attack can be easily observed. When increased the AOA value the lift coefficient was also increase. At approximately $14^{\circ}$ the airfoil showed it was stalled. Stall is the condition where upper surface flow is more separated and the airfoil or blade is generating its maximum lift coefficient. In Figure 10(b) the drag coefficient is decreasing gradually when angle of attack increases. The drag coefficient was maximum for high angle of attack and low for low angle of attack. The graph shows the drag coefficient increases when the angle of attack increases because the frontal area of the wing increases along the wind direction as we know that the drag force is the resistive force so the drag coefficient increases gradually. In Figure 10 (c) the AOA and $C_{L} / C_{D}$ ratio can be observed. The graph shows that at $2^{\circ}$ and $4^{\circ}$ the lift to drag ratio is not decreased because in the table 4.1 at angle $2^{\circ}$ and $4^{\circ}$ when the lift coefficient increased the drag coefficient is not increased so the ratio was not changed. Therefore there is a constant behavior shown at $2^{\circ}$ and $4^{\circ}$.

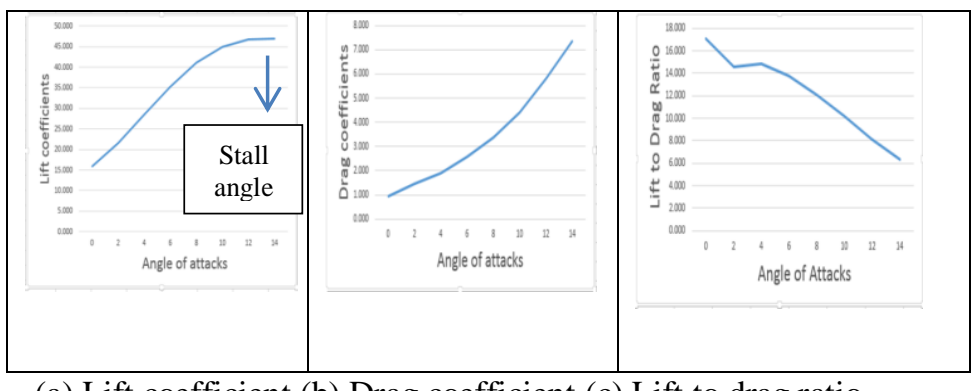

(a) Lift coefficient (b) Drag coefficient (c) Lift to drag ratio

Figure 10: Coefficients against angle of attack 


\subsection{Pressure and velocity contours}

\subsubsection{Pressure and velocity contour at $2^{\circ}$ Angle of attack}

In Figure 11 (a) and (b) the two dimensional contours of the coefficient of pressure and coefficient of velocity is shown below the streamlines are surrounded the airfoil for the angle of attack of $2^{\circ}$. When pressure increases on the wing the velocity decreases on the same time. (a) Pressure contour at $2^{\circ}$

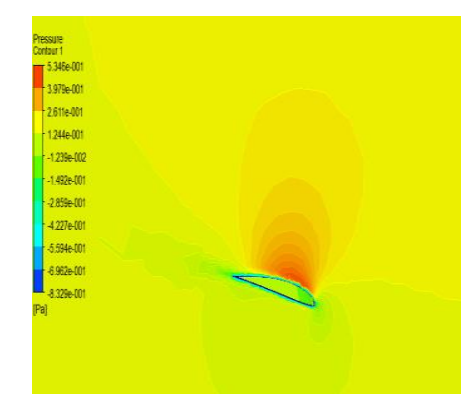

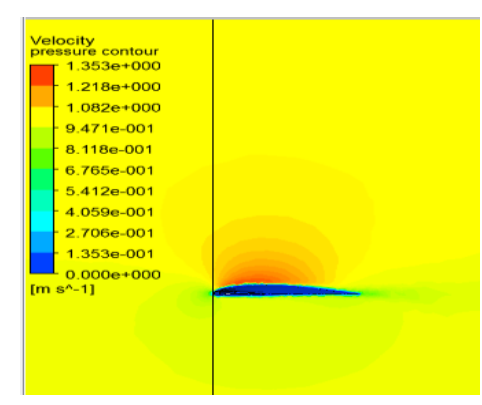

(b) Velocity contour at $2^{\circ}$
Figure 11: Pressure and velocity contours

\subsubsection{Pressure contour at $4 \circ$ Angle of attack}

In Figure 12 (a) and (b) the two dimensional contours of the coefficient of pressure and coefficient of velocity is shown below the streamlines are surrounded the airfoil for the angle of attack of $4^{\circ}$. When pressure increases on the wing the velocity decreases on the same time.

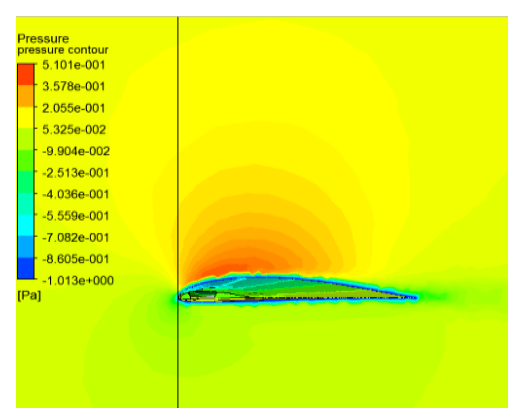

(a) Pressure Contour at $4^{\circ}$

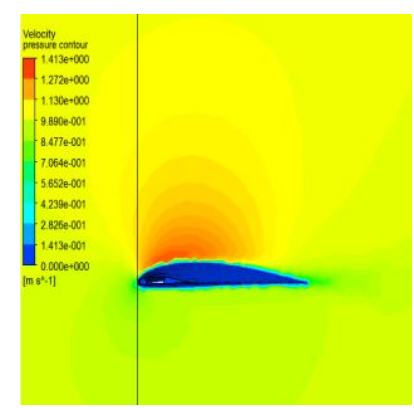

(b) Velocity contour at $4^{\circ}$
Figure 12: Pressure and velocity contours

\subsection{Smart Structures}

In this project the second aim was to apply a smart structure on the blade which is designed earlier in Section 3.2.

\subsubsection{Active Twist}

The blade airfoil and geometry is same and the computational domain is also same. In geometry, the tip of the blade is twisted at different angles i.e. $0^{\circ}, 2^{\circ}, 4^{\circ}, 6^{\circ}, 8^{\circ}$ and $10^{\circ}$. Positive twist angles given were used, i.e. blade tip is rotated anticlockwise. In Mesh, the element size were similar as discussed earlier in Section 3.4.The turbulence model $k-\varepsilon$ was used, with same boundary conditions as discussed earlier.

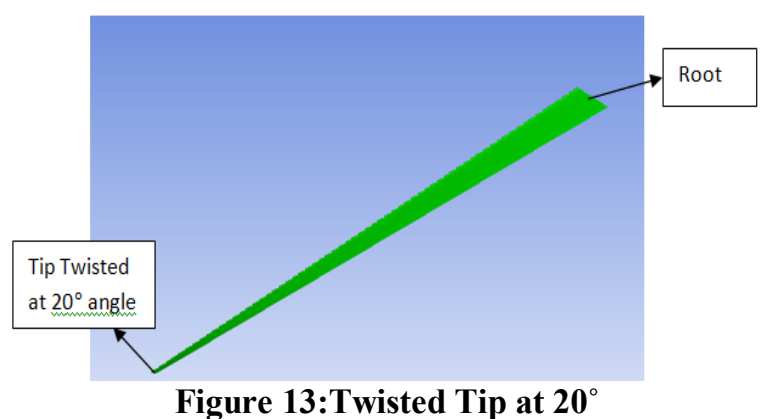

Figure 13:Twisted Tip at $20^{\circ}$
4.3.2Twist Angle values at $0^{\circ}$ Angle of attack

In table 4.2 , there are different $C_{L} / C_{D}$ ratio values at different twist angles from $0^{\circ}-10^{\circ}$.In the table maximum $\frac{C_{L}}{C_{D}}$ is shown from $4^{\circ}-8^{\circ}$.At these angles the blade can perform better due to the high ratio of $C_{L}$ and $C_{D}$. As we know that the blade twist maximizes the angle of attack along the length of 


\section{International Journal of Engineering Applied Sciences and Technology, 2021 \\ Vol. 5, Issue 11, ISSN No. 2455-2143, Pages 62-71 \\ Published Online March 2021 in IJEAST (http://www.ijeast.com)}

the blade to get the best lift and rotation. The purpose of this research is to design a blade of high lift coefficient so that the blade efficiency will increase and we can see that at $4^{\circ}-8^{\circ}$ twist angle the ratio of $\frac{C_{L}}{C_{D}}$ is maximum so the twisted blade will be performed better at these angle with comparison to untwisted blade.

\begin{tabular}{|c|c|c|c|c|}
\hline $\begin{array}{c}\text { Twist } \\
\left.\text { Angle [ }{ }^{\circ}\right]\end{array}$ & $\begin{array}{c}\text { Angle of } \\
\left.\text { Attack [ }{ }^{\circ}\right]\end{array}$ & $\mathbf{C}_{\mathbf{L}}$ & $\mathbf{C}_{\mathbf{D}}$ & $\mathbf{C}_{\mathbf{L}} / \mathbf{C}_{\mathbf{D}}$ \\
\hline $\mathbf{0}$ & 0 & 15.934 & 0.93276 & 17.08 \\
\hline $\mathbf{2}$ & 0 & 9.125 & 0.7654 & 11.96 \\
\hline $\mathbf{4}$ & 0 & 17.812 & 0.6632 & 26.8 \\
\hline $\mathbf{6}$ & 0 & 19.543 & 0.5789 & 33.8 \\
\hline $\mathbf{8}$ & 0 & 21.012 & 0.5424 & 38.9 \\
\hline $\mathbf{1 0}$ & 0 & 17.945 & 0.6990 & 25.27 \\
\hline
\end{tabular}

Table 4.2:- $C_{L}, C_{D}$ and $C_{L} / C_{D}$ values for change in angle of twist of blade for $0^{\circ}$ angle of attack

\subsubsection{Effect of twist angle on lift coefficient}

In Figure 14(a) the effect of twist angle on the lift coefficient is shown. In graph shows that at $4^{\circ}-8^{\circ}$ the maximum increase in lift coefficient. In Figure 14(b) the effect of twist angle on drag coefficient is shown. In Figure 14(c) the effect of twist angle on lift / drag ratio is shown. The graph shows the lift to drag ratio is maximum at $4^{\circ}-8^{\circ}$.
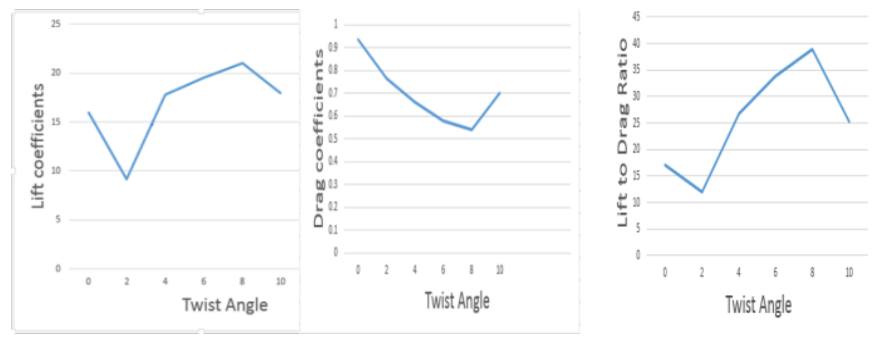

(a)Lift coefficient (b) Drag coefficient (c) Lift to drag ratio

Figure 14: Twist angle against coefficients

\section{CONCLUSIONS}

This study investigated the effect of smart structures on aerodynamic performance of a horizontal axis wind turbine. The CFD simulation has proven for this particular airfoil S4310 that the most effective angle of attack is $12^{\circ}$. The maximum increase in lift coefficient was between $8^{\circ}-14^{\circ}$.In other words, within this range there will be effective use of the airfoil.

In active twist the blade efficiency will be greater at angle $4^{\circ}$ $8^{\circ}$ due to high $C_{L} / C_{D}$ ratio. The twisted blade is better than from untwisted blade because when the twist is given to the blade the direction of wind is also change so it effects the lift force and the lift force increases so the efficiency will also increase. For calculating the lift and drag coefficient, particularly for high angle of attack the ANSYS FLUENT is a good software.

Using the $k-\varepsilon$ turbulence model, it has been observed that the fluent shows the good result at inlet velocity which is 9 $\mathrm{m} / \mathrm{s}$. CFD is good to calculate the best and acute angle of attack. The CFD software used in this project has some limitations when the mesh portion was performed the solution of this problem is that for meshing use the different software which is only specific for meshing or used another software which have more mesh options. For CFD simulation high speed computers should be used for better result. For future work the active twist blade can be also performing on a different angle of attack and also calculate the aerodynamic forces. Due to the time limitation the smart structures i.e. active flap and active tip cannot be investigated so in future that work can be performed.

\section{REFERENCES}

[1] R.Ryoichi,"Horizontal axis wind turbine blade design",2009.

[2] HanCao, "Aerodynamic Analysis of small horizontal axis wind turbine blades by using 2D and 3D CFD modelling". England,2011.

[3] Hansen. "2-D Aerodynamics. In Hansen, Aerodynamic of wind turbine" 2008,UK: Earthscan.

[4] J.F.Manwell, J. \& ,"Wind energy explained Theory design and application”,2009 ,London: John Wiley \& sons Ltd

[5] L.Tangler, "The Evolution of rotor and blade design. American Wind energy association",2000, California

[6] Peter J.Schubel, R. J. ,"Wind turbine blade design. Energies, 2012.

"[7] K.Y.Maalawi, M. B. , "A practical approach for selecting optimum wind rotors", Renewable energy, 803-822.

[8] T.K. Barlas, G. "Review of the state of the art in smart rotor control research for wind turbines". Aerospace secience, $1-27,2003$.

[9] K.Mcwilliam, M. "Aeroelastic wind turbine design with active flaps for AEP maximization”,DTU wind energy,2018. 
[10] UIUC airfoil database. (n.d.). Retrieved from UIUC airfoil database site: http://m-seling.ae.illinois.edu.html

[11] ] M.Grujicic, G. A. "Structural response analysis of 1 MW horizontal axis wind turbine". JMEPEG , 790-801,2010.

[12] "Investigation on wind power potential of karachi. karachi”.2000-2005. 